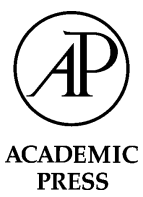

PRESS

\title{
The value of public information in a Cournot duopoly
}

\author{
Ezra Einy, ${ }^{\mathrm{a}}$ Diego Moreno, ${ }^{\mathrm{b}, *}$ and Benyamin Shitovitz ${ }^{\mathrm{c}}$ \\ a Department of Economics, Ben Gurion University, PO BOX 653, Beer-Sheva 84105, Israel \\ b Departamento de Economía, Universidad Carlos III de Madrid, 28903 Getafe, Spain \\ c Department of Economics, University of Haifa, Mount Carmel, Haifa 31905, Israel
}

Received 25 March 2002

\begin{abstract}
We derive alternative sufficient conditions for the value of public information to be either positive or negative in a Cournot duopoly where firms technology exhibits constant returns to scale. (c) 2003 Elsevier Inc. All rights reserved.

JEL classification: C72; D43; L13
\end{abstract}

\section{Introduction}

The purpose of this paper is to study the value of public information in a Cournot duopoly where there is uncertainty about the market demand and/or the cost function. We provide conditions that allow one to determine whether the value of public information is positive or negative. With every Cournot duopoly in a certain class, we associate a real-valued function (defined on a convex subset of the positive orthant of the real plane) whose curvature determines whether the value of public information is either positive or negative: if this function is convex (concave) then the value of public information is positive (negative). Using this fact we identified interesting subclasses of industries where the value of public information is positive (negative). We also show the usefulness of our results to determine the value of public information in specific applications.

There is a considerable literature studying the value of public information in general equilibrium. Hirshleifer (1971) shows that improving public information may make agents

\footnotetext{
* Corresponding author.

E-mail address: dmoreno@eco.uc3m.es (D. Moreno).
} 
worse off ex-ante in an exchange economy where agents share risks. Several papers generalize aspects of Hirshleifer examples—see, e.g., Marshall (1979), Wilson (1975), Green (1981), Sulganik and Zilcha (1996). In a recent paper, Schlee (2001) shows that in this context the value of public information is negative in any economy where there is a representative agent. Contrary to Schlee's result, in our context we can easily generate examples of classes of industries for which the value of public information is positive.

There are also a number of papers that study the value of information in a linear oligopoly. ${ }^{1}$ Ponssard (1979) investigates this issue in an industry where there is uncertainty about the market demand, and where some firms are informed about the state of nature and other are uninformed. Vives (1984) studies the value of information under both Cournot and Bertrand competition in a duopoly where demand is uncertain. Also in a linear duopoly where firms are uncertain about their costs, Sakai (1985) investigates the value of information under a variety of information structures.

In studying the value of information in a Cournot oligopoly some difficulties emerge. Specifically, the associated non-cooperative game with incomplete information may not have a unique and/or interior equilibrium. When the game has several equilibrium points, it is not clear which equilibria to compare. And when equilibrium is not interior, comparative static exercises are difficult as corner equilibria are characterized by a set of inequalities rather than a system of equations. Moreover, it is easy to produce examples of industries whose associated game has a unique and interior equilibrium, for which altering the firms' information structure by adding public information leads to a new game whose unique equilibrium is a corner equilibrium (see Example 4.1 below).

All the papers mentioned above circumvent this problem by directly assuming that the games associated with the industries under study have a unique and interior equilibrium, even though it is not difficult to find examples where this assumption is violated. Instead, we identify a class of Cournot duopolies (not necessarily linear) with symmetric information for which the game associated to each industry has a unique interior equilibrium. This allows us to define the value of public information for any industry in this class, and study conditions under which it is either positive or negative. In addition, our model of incomplete information does not impose any restriction of the space of states of nature or on the character of firms' information. In particular, our framework allows for continuous as well as discrete information structures.

There are other topics on information in oligopolistic environments that have received attention in the literature. Gal-Or $(1985,1986)$, for example, studies the incentives for information sharing, and Einy et al. (2002) examine whether information advantages are rewarded in equilibrium. Studying these issues involves exercises different from those performed in the present paper. Determining whether a firm may have an incentive to reveal (part or all of) its information to a rival, for example, requires to compare the payoffs of the firm in two games that differ in the information of the rival. Or determining whether a firm with superior information enjoys greater profits requires to compare the profits of the firms in a (given) game. Our results offer no conclusion regarding these issues.

\footnotetext{
${ }^{1}$ In a recent paper, Ottaviani and Pratt (2001) study the value of public information in a monopoly.
} 


\section{The model}

Consider a duopolistic industry where two firms compete in the production of a homogeneous good. There is uncertainty about the industry's demand and the firms' costs. This uncertainty is described by a probability space $(\Omega, \mathcal{F}, \mu)$, where $\Omega$ is the set of states of nature, $\mathcal{F}$ is a $\sigma$-field of subsets of $\Omega$, and $\mu$ is a $\sigma$-additive probability measure on $(\Omega, \mathcal{F})$. (We interpret $\mu$ as the common prior of the firms.) Once the state of nature $\omega \in \Omega$ is realized, the market demand, and the firms' costs are determined. Write $p: \Omega \times \mathbb{R}_{+} \rightarrow \mathbb{R}_{+}$for the inverse market demand function, and for $i \in\{1,2\}$ write $c_{i}: \Omega \times \mathbb{R}_{+} \rightarrow \mathbb{R}$ for Firm $i$ 's cost function. The information of Firm $i \in\{1,2\}$ about the state of nature is described by a $\sigma$-subfield $\mathcal{F}_{i}$ of $\mathcal{F}$; that is, given an event $A \in \mathcal{F}_{i}$, Firm $i$ knows whether the realized state of nature is a member of $A$. We refer to $\mathcal{F}_{i}$ as Firm $i$ 's information field. A duopolistic industry with incomplete information is thus described by a collection $I=\left((\Omega, \mathcal{F}, \mu), p, c_{1}, c_{2}, \mathcal{F}_{1}, \mathcal{F}_{2}\right)$.

Throughout the paper we assume that the inverse demand function, $p$, and the cost functions, $c_{1}$ and $c_{2}$, of any duopolistic industry with incomplete information are such that for every integrable function $q$, the functions $q p(\cdot, q(\cdot)), c_{1}(\cdot, q(\cdot))$, and $c_{2}(\cdot, q(\cdot))$ are also integrable.

Let $I$ be a duopolistic industry with differential information. The Bayesian game associated with $I$ is the collection $G(I)=\left((\Omega, \mathcal{F}, \mu), \mathbb{R}_{+}^{2},\left(\mathcal{F}_{1}, \mathcal{F}_{2}\right),\left(\pi_{1}, \pi_{2}\right)\right)$, where for each firm $i \in\{1,2\}$ the set of possible actions is $\mathbb{R}_{+}$, and its profit function $\pi_{i}: \Omega \times \mathbb{R}_{+}^{2} \rightarrow \mathbb{R}$ is given for all $\omega \in \Omega$ and $r=\left(r_{1}, r_{2}\right) \in \mathbb{R}_{+}^{2}$ by

$$
\pi_{i}(\omega, r)=r_{i} p\left(\omega, r_{1}+r_{2}\right)-c_{i}\left(\omega, r_{i}\right) .
$$

We refer to $G(I)$ as the Cournot game with incomplete information associated with the industry $I$. In this game, a (pure) strategy for a firm $i \in\{1,2\}$ is an $\mathcal{F}_{i}$-measurable function $q_{i}: \Omega \rightarrow \mathbb{R}_{+}$whose first and second moments exist. We denote by $S_{i}$ the set of all strategies for Firm $i$, and by $S=S_{1} \times S_{2}$ the set of profiles of strategies.

Let $X$ be an integrable random variable on $(\Omega, \mathcal{F}, \mu)$, and let $\mathcal{G}$ be a $\sigma$-subfield of $\mathcal{F}$. We write $E(X \mid \mathcal{G})$ for the conditional expectation of $X$ with respect to $\mathcal{G}$. Let $G(I)$ be a Cournot game with incomplete information. A Bayesian equilibrium is a profile of strategies $q^{*}=\left(q_{1}^{*}, q_{2}^{*}\right) \in S$ such that for every $i \in\{1,2\}$ and every $q_{i} \in S_{i}$,

$$
E\left(\pi_{i}\left(\cdot, q^{*}(\cdot)\right) \mid \mathcal{F}_{i}\right)(\omega) \geqslant E\left(\pi_{i}\left(\cdot,\left(q_{i}(\cdot), q_{-i}^{*}(\cdot)\right)\right) \mid \mathcal{F}_{i}\right)(\omega),
$$

for almost every $\omega \in \Omega$. (Our assumptions on $p, c_{1}, c_{2}$ and on the set of strategies of every firm guarantee that for all $i \in\{1,2\}$ and $q \in S$, and for every $\sigma$-subfield $\mathcal{G}$ of $\mathcal{F}$, $E\left(\pi_{i}(\cdot, q(\cdot)) \mid \mathcal{G}\right)$ exists. $)$

Remark 2.1. Equilibrium condition (2.1) requires that at a Bayesian equilibrium every firm maximizes its (interim) conditional expected profits at every state of nature. This condition is equivalent to requiring that each firm maximizes its ex-ante expected profits; i.e., condition (2.1) is equivalent to

$$
E\left(\pi_{i}\left(\cdot, q^{*}(\cdot)\right)\right) \geqslant E\left(\pi_{i}\left(\cdot,\left(q_{i}(\cdot), q_{-i}^{*}(\cdot)\right)\right)\right),
$$

for every $i \in\{1,2\}$ and every $q_{i} \in S_{i}$. 
Proof. Clearly (2.1) implies (2.2). To prove the converse, assume that (2.1) does not hold; then there is $i \in\{1,2\}$ and $q_{i} \in S_{i}$ such that

$$
E\left(\pi_{i}\left(\cdot, q_{i}(\cdot), q_{-i}^{*}(\cdot)\right) \mid \mathcal{F}_{i}\right)>E\left(\pi_{i}\left(\cdot, q^{*}(\cdot)\right) \mid \mathcal{F}_{i}\right)
$$

on some event $A \in \mathcal{F}_{i}$ with $\mu(A)>0$. Define $\hat{q}_{i}: \Omega \rightarrow \mathbb{R}_{+}$by

$$
\hat{q}_{i}(\omega)= \begin{cases}q_{i}(\omega) & \text { if } \omega \in A, \\ q_{i}^{*}(\omega) & \text { if } \omega \in \Omega \backslash A .\end{cases}
$$

Then $\hat{q}_{i} \in S_{i}$ and

$$
\begin{aligned}
E & \left(\pi_{i}\left(\cdot, \hat{q}_{i}(\cdot), q_{-i}^{*}(\cdot)\right)\right) \\
& =\int_{\Omega} \pi_{i}\left(\cdot, \hat{q}_{i}(\cdot), q_{-i}^{*}(\cdot)\right) \mathrm{d} \mu \\
& =\int_{A} \pi_{i}\left(\cdot, \hat{q}_{i}(\cdot), q_{-i}^{*}(\cdot)\right) \mathrm{d} \mu+\int_{\Omega \backslash A} \pi_{i}\left(\cdot, \hat{q}_{i}(\cdot), q_{-i}^{*}(\cdot)\right) \mathrm{d} \mu \\
& =\int_{A} E\left(\pi_{i}\left(\cdot, q_{i}(\cdot), q_{-i}^{*}(\cdot)\right) \mid \mathcal{F}_{i}\right) \mathrm{d} \mu+\int_{\Omega \backslash A} \pi_{i}\left(\cdot, q_{i}^{*}(\cdot), q_{-i}^{*}(\cdot)\right) \mathrm{d} \mu \\
& >E\left(\pi_{i}\left(\cdot, q^{*}(\cdot)\right)\right) .
\end{aligned}
$$

Thus, (2.2) does not hold.

\section{The value of public information}

In this section we study the value of public information in a symmetric duopoly; i.e., in an industry $I$ where both firms have identical information (i.e., $\mathcal{F}_{1}=\mathcal{F}_{2}=\mathcal{G}$ ) and cost (i.e., $\left.c_{1}=c_{2}=c\right)$. Thus, a symmetric duopolistic industry $I$ can be described by a collection $((\Omega, \mathcal{F}, \mu), p, c, \mathcal{G})$. (For economy of notation we do not repeat $c$ and $\mathcal{G}$.) We refer to the game $G(I)$ associated to a symmetric duopolistic industry as a Cournot game with symmetric information.

Theorem 3.1 provides conditions on the demand and cost functions that guarantee existence, uniqueness, symmetry and interiority of Bayesian equilibria in a Cournot game with symmetric information. Note the "multiplicative" nature of the uncertainty in demand and cost assumed in Theorem 3.1. The proof of Theorem 3.1 relies on Amir (1996) for existence and uniqueness, although in order to guarantee the measurability of the equilibrium strategies we have to appeal to Aumann's Measurable Selection Theorem-see Aumann (1969). For interiority and symmetry we provide an argument based on first-order conditions for profit maximization.

Theorem 3.1. Let $I=((\Omega, \mathcal{F}, \mu), p, c, \mathcal{G})$ be a symmetric duopolistic industry. Assume that for all $(\omega, x) \in \Omega \times \mathbb{R}_{+}, \quad p(\omega, x)=\alpha(\omega) f(x)$ and $c(\omega, x)=\beta(\omega) x$, where $\alpha, \beta: \Omega \rightarrow \mathbb{R}_{++}$are integrable functions and $f: \mathbb{R}_{+} \rightarrow \mathbb{R}_{+}$satisfies 
(3.1.1) there is $\bar{x} \in \mathbb{R}_{+}$such that $f(x)>0$ for $x \in[0, \bar{x})$, and $f(x)=0$ for $x \geqslant \bar{x}$;

(3.1.2) $f$ is differentiable and strictly decreasing on $[0, \bar{x})$;

(3.1.3) $f$ is log concave on $[0, \bar{x})$; and

(3.1.4) $\alpha(\omega) f(0)>\beta(\omega)>0$ for all $\omega \in \Omega$.

Then $G(I)$ has a unique Bayesian equilibrium $\left(q_{1}^{*}, q_{2}^{*}\right)$. Moreover, $q_{1}^{*}(\omega)=q_{2}^{*}(\omega) \in$ $(0, \bar{x} / 2)$ for all $\omega \in \Omega$.

Proof. For every $\omega \in \Omega$ define the two-player game of complete information $G(\omega, I)$ where each player $i \in\{1,2\}$ set of pure strategies is $\mathbb{R}_{+}$, and its payoff function $\sigma_{i}(\omega, \cdot): \mathbb{R}_{+}^{2} \rightarrow \mathbb{R}_{+}$is given by

$$
\sigma_{i}(\omega,(x, y))=E\left(\pi_{i}(\cdot,(x, y)) \mid \mathcal{G}\right)(\omega),
$$

where

$$
\pi_{1}(\cdot,(x, y))=x p(\cdot,(x+y))-c(\cdot, x)
$$

and

$$
\pi_{2}(\cdot,(x, y))=y p(\cdot,(x+y))-c(\cdot, y) .
$$

It is easy to check that under assumptions (3.1.1)-(3.1.4) the game $G(\omega, I)$ satisfies the assumptions of Theorem 2.3 of Amir (1996) and therefore has a unique Nash equilibrium, $\left(q_{1}^{*}(\omega), q_{2}^{*}(\omega)\right)$. We show that $q^{*}(\cdot)=\left(q_{1}^{*}(\cdot), q_{2}^{*}(\cdot)\right)$ is a Bayesian equilibrium of $G(I)$. We first show that $q^{*}(\cdot)$ is a $\mathcal{G}$-measurable function. Define the correspondence $E: \Omega \rightarrow 2^{\mathbb{R}_{+}}$ by

$$
E(\omega)=\left\{(x, y) \in \mathbb{R}_{+}^{2} \mid(x, y) \text { is a Nash equilibrium of } G(\omega, I)\right\} .
$$

We show that the graph of the correspondence $E$ is measurable with respect to the product $\sigma$-field $\mathcal{G} \otimes \mathcal{B}\left(\mathbb{R}_{+}^{2}\right)$, where $\mathcal{B}\left(\mathbb{R}_{+}^{2}\right)$ is the $\sigma$-field of Borel subsets of $\mathbb{R}_{+}^{2}$. For all $(a, b) \in \mathbb{R}_{+}^{2}$ let $D(a, b)$ be the set

$$
\begin{aligned}
\left\{(\omega,(x, y)) \in \Omega \times \mathbb{R}_{+}^{2} \mid \sigma_{1}(\omega,(x, y))\right. & \geqslant \sigma_{1}(\omega,(a, y)) \text { and } \\
\sigma_{2}(\omega,(x, y)) & \left.\geqslant \sigma_{2}(\omega,(x, b))\right\} .
\end{aligned}
$$

Since for all $(x, y) \in \mathbb{R}_{+}^{2}$ the functions $\sigma_{1}(\cdot,(x, y))$ and $\sigma_{2}(\cdot,(x, y))$ are $\mathcal{G}$-measurable, and for all $\omega \in \Omega$ the functions $\sigma_{1}(\omega, \cdot)$ and $\sigma_{2}(\omega, \cdot)$ are continuous in $\mathbb{R}_{+}^{2}$, then for all $(a, b) \in \mathbb{R}_{+}^{2}$ the set $D(a, b)$ is $\mathcal{G} \otimes \mathcal{B}\left(\mathbb{R}_{+}^{2}\right)$-measurable. Now, the graph of $E$ is given by

$$
\operatorname{graph}(E)=\bigcap_{(a, b) \in \mathbb{R}_{+}^{2}} D(a, b)=\bigcap_{(a, b) \in \mathbb{Q}_{+}^{2}} D(a, b),
$$

where $\mathbb{Q}_{+}^{2}$ denotes the set of duples of non-negative rational numbers. Since the set $\mathbb{Q}_{+}^{2}$ is countable, $\operatorname{graph}(E)$ is $\mathcal{G} \otimes \mathcal{B}\left(\mathbb{R}_{+}^{2}\right)$-measurable. Thus, by the Measurable Selection Theorem (see Aumann (1969) and Hildenbrand (1974, Theorem 1 on p. 54)), there exists a $\mathcal{G}$-measurable function $\phi: \Omega \rightarrow \mathbb{R}^{2}$ such that $\phi(\omega) \in E(\omega)$ for almost all $\omega \in \Omega$. Since for all $\omega \in \Omega$ the set $E(\omega)$ is a singleton (because $G(\omega, I)$ has a unique equilibrium), 
$\phi(\omega)=q^{*}(\omega)$ for almost all $\omega \in \Omega$, and therefore $q^{*}$ is a $\mathcal{G}$-measurable function. Now, for each $i \in\{1,2\}$ and $q_{i} \in S_{i}$ we have

$$
E\left(\pi_{i}\left(\cdot, q^{*}(\omega)\right) \mid \mathcal{G}\right)(\omega) \geqslant E\left(\pi_{i}\left(\cdot,\left(q_{i}(\omega), q_{-i}^{*}(\omega)\right)\right) \mid \mathcal{G}\right)(\omega)
$$

for every $\omega \in \Omega$, and therefore

$$
E\left(\pi_{i}\left(\cdot, q^{*}(\cdot)\right)\right) \geqslant E\left(\pi_{i}\left(\cdot,\left(q_{i}(\cdot), q_{-i}^{*}(\cdot)\right)\right)\right),
$$

which by Remark 2.1 establishes that $q^{*}$ is an Bayesian equilibrium of $G(I)$.

Uniqueness of $q^{*}$ follows from the fact that for all $\omega \in \Omega$ the game $G(\omega, I)$ has a unique equilibrium.

It remains to show that for all $\omega \in \Omega, q_{1}^{*}(\omega)=q_{2}^{*}(\omega) \in(0, \bar{x} / 2)$. Let $\omega \in \Omega$. We first show that $q_{1}^{*}(\omega), q_{2}^{*}(\omega) \in(0, \bar{x})$. If $q_{i}^{*}(\omega) \geqslant \bar{x}$ for some $i \in\{1,2\}$, then $p\left(\cdot, q_{1}^{*}(\omega)+q_{2}^{*}(\omega)\right)=0$, and therefore $\sigma_{i}\left(\omega,\left(q_{1}(\omega), q_{2}(\omega)\right)<0\right.$, which contradicts that $\left(q_{1}^{*}(\omega), q_{2}^{*}(\omega)\right)$ is an equilibrium of $G(\omega, I)$ (because a Firm $i$ can guarantee itself zero profits by producing $\left.q_{i}(\omega)=0\right)$. Thus $q_{i}^{*}(\omega)<\bar{x}$ for all $i \in\{1,2\}$. We show that $q_{i}^{*}(\omega)>0$ for all $i \in\{1,2\}$. Assume by way of contradiction that $q_{i}^{*}(\omega)=0$ for some $i \in\{1,2\}$. Without loss of generality set $i=1$. The Kuhn-Tucker condition for profit maximization implies

$$
E\left(\left(p\left(\cdot, q_{2}^{*}(\omega)\right)-\beta(\cdot)\right) \mid \mathcal{G}\right)(\omega) \leqslant 0 .
$$

If $q_{2}^{*}(\omega)=0$, then by (3.1) we have

$$
E(p(\cdot, 0) \mid \mathcal{G})(\omega) \leqslant E(\beta(\cdot) \mid \mathcal{G})(\omega),
$$

which contradicts condition (3.1.4). If $\bar{x}>q_{2}^{*}(\omega)>0$, then Firm 2's first-order condition for profit maximization implies

$$
E\left(q_{2}^{*}(\omega) p^{\prime}\left(\cdot, q_{2}^{*}(\omega)\right)+p\left(\cdot, q_{2}^{*}(\omega)\right) \mid \mathcal{G}\right)(\omega)=E(\beta(\cdot) \mid \mathcal{G})(\omega) .
$$

And since $p^{\prime}\left(\cdot, q_{2}^{*}(\omega)\right)<0$ and $q_{2}^{*}(\omega)>0$, we have

$$
E\left(p\left(\cdot, q_{2}^{*}(\omega)\right) \mid \mathcal{G}\right)(\omega)>E(\beta(\cdot) \mid \mathcal{G})(\omega),
$$

which contradicts (3.1).

Finally we show that $q_{1}^{*}(\omega)=q_{2}^{*}(\omega)<\bar{x} / 2$. Since $0<q_{i}^{*}(\omega)<\bar{x}$ for all $i \in\{1,2\}$, the first-order conditions for profits maximization imply

$$
E\left(q_{i}^{*}(\omega) p^{\prime}\left(\cdot, q_{1}^{*}(\omega)+q_{2}^{*}(\omega)\right)+p\left(\cdot, q_{1}^{*}(\omega)+q_{2}^{*}(\omega)\right) \mid \mathcal{G}\right)(\omega)=E(\beta(\cdot) \mid \mathcal{G})(\omega)
$$

for all $i \in\{1,2\}$. Therefore

$$
E\left(q_{1}^{*}(\omega) p^{\prime}\left(\cdot, q_{1}^{*}(\omega)+q_{2}^{*}(\omega)\right) \mid \mathcal{G}\right)(\omega)=E\left(q_{2}^{*}(\omega) p^{\prime}\left(\cdot, q_{1}^{*}(\omega)+q_{2}^{*}(\omega)\right) \mid \mathcal{G}\right)(\omega),
$$

and since $\left(q_{1}^{*}(\omega), q_{2}^{*}(\omega)\right)$ is a Nash equilibrium of $G(\omega, I)$, we have $q_{1}^{*}(\omega)+q_{2}^{*}(\omega)<\bar{x}$. Hence $p^{\prime}\left(\cdot, q_{1}^{*}(\omega)+q_{2}^{*}(\omega)\right)<0$, and therefore $q_{1}^{*}(\omega)=q_{2}^{*}(\omega)<\bar{x} / 2$.

Throughout the rest of the section let us be given a probability space $(\Omega, \mathcal{F}, \mu)$. A symmetric duopolistic industry $I$ is thus described by a demand and a cost function, and a $\sigma$-subfield $\mathcal{G}$ of $\mathcal{F}$. Given a market demand $p$ and a cost function $c$, define the binary 
relation $\succeq$ on the family of all $\sigma$-subfields of $\mathcal{F}$ as follows: If $\mathcal{G}$ and $\mathcal{H}$ are two $\sigma$-subfields of $\mathcal{F}$, then

$$
\mathcal{H} \succeq \mathcal{G} \quad \Leftrightarrow \quad\left\{\begin{array}{l}
E(p(\cdot, x) \mid \mathcal{H})=E(p(\cdot, x) \mid \mathcal{G} \vee \mathcal{H}) \quad \text { and } \\
E(c(\cdot, x) \mid \mathcal{H})=E(c(\cdot, x) \mid \mathcal{G} \vee \mathcal{H}) \quad \forall x \in \mathbb{R}
\end{array}\right\} .
$$

(Here $\mathcal{G} \vee \mathcal{H}$ is the smallest $\sigma$-subfield of $\mathcal{F}$ that contains both $\mathcal{G}$ and $\mathcal{H}$.)

The interpretation of the binary relation $\succeq$ is simple: $\mathcal{H} \succeq \mathcal{G}$ if (and only if) the predictions of demand and cost functions (the uncertain parameters of the industry) are the same whether the firms information is given by $\mathcal{H}$, or by the aggregate information in $\mathcal{G}$ and $\mathcal{H}$ (i.e., by $\mathcal{G} \vee \mathcal{H}$ ). The binary relation $\succeq$ contains that introduced in Blackwell (1951); i.e., if $\mathcal{H}$ is more valuable than $\mathcal{G}$ in the sense of Blackwell (1951), then $\mathcal{H} \succeq \mathcal{G}$, but the converse may not hold: If $\mathcal{H}$ and $\mathcal{G}$ are generated by finite partitions of $\Omega$, for example, then $\mathcal{H}$ is more valuable in the sense of Blackwell than $\mathcal{G}$ if and only if $\mathcal{H} \supseteq \mathcal{G}$-see Laffont (1989, Theorem 1 in Chapter 4). It is clear that $\mathcal{H} \supseteq \mathcal{G}$ implies $\mathcal{H} \succeq \mathcal{G}$, and therefore that $\succeq$ contains Blackwell's relation. However, it is easy to construct an example for which $\mathcal{H} \succeq \mathcal{G}$ even though $\mathcal{H} \nsupseteq \mathcal{G}$ - for an example of this kind, see Example 1 in Einy et al. (2002).

Now, consider an industry where the market demand is given for $(\omega, x) \in \Omega \times \mathbb{R}_{+}$by $p(\omega, x)=\alpha(\omega) f(x)$, and where firms' cost is $c(\omega, x)=\beta(\omega) x$, where $\alpha, \beta: \Omega \rightarrow \mathbb{R}$ are $\mathcal{F}$-measurable integrable functions. Then

$$
\mathcal{H} \succeq \mathcal{G} \quad \Leftrightarrow \quad\{E(\alpha \mid \mathcal{H})=E(\alpha \mid \mathcal{G} \vee \mathcal{H}) \text { and } E(\beta \mid \mathcal{H})=E(\beta \mid \mathcal{G} \vee \mathcal{H})\}
$$

Let $p: \Omega \times \mathbb{R}_{+} \rightarrow \mathbb{R}$ and $c: \Omega \times \mathbb{R}_{+} \rightarrow \mathbb{R}$ be given for $(\omega, x) \in \Omega \times \mathbb{R}_{+}$by $p(\omega, x)=\alpha(\omega) f(x)$, and $c(\omega, x)=\beta(\omega) x$, where $\alpha, \beta$ and $f$ satisfy the assumptions of Theorem 3.1. For every $\sigma$-subfield $\mathcal{G}$ of $\mathcal{F}$ consider the symmetric duopolistic industry $I=((\Omega, \mathcal{F}, \mu), p, c, \mathcal{G})$. By Theorem 3.1, the Cournot game $G(I)$ has a unique Bayesian equilibrium, which is symmetric. Denote this equilibrium by $\left(q_{\mathcal{G}}^{*}, q_{\mathcal{G}}^{*}\right)$, and the equilibrium profit by $\pi_{\mathcal{G}}^{*}$. We say that the value of public information in the industry I is positive (negative) if for every $\sigma$-subfield $\mathcal{H}$ of $\mathcal{F}$

$$
\mathcal{H} \succeq \mathcal{G} \Rightarrow E\left(\pi_{\mathcal{H}}^{*}\right) \geqslant E\left(\pi_{\mathcal{G}}^{*}\right)\left(E\left(\pi_{\mathcal{H}}^{*}\right) \leqslant E\left(\pi_{\mathcal{G}}^{*}\right)\right)
$$

That is, the value of public information is positive (negative) if having better information does not decrease (increase) firms' expected profits.

Let $f: \mathbb{R}_{+} \rightarrow \mathbb{R}_{+}$be a function satisfying the assumptions (3.1.1)-(3.1.3) of Theorem 3.1. Define

$$
K(f)=\left\{(\alpha, \beta) \mid \alpha, \beta: \Omega \rightarrow \mathbb{R}_{++} \text {are integrable and } f(0) \alpha(\omega)>\beta(\omega) \forall \omega \in \Omega\right\} .
$$

Note that $K(f)$ is a convex subset of $L_{1}(\Omega, \mathcal{F}, \mu) \times L_{1}(\Omega, \mathcal{F}, \mu)$. We denote by $\mathcal{I}(f)$ the class of duopolistic industries of the form $I=((\Omega, \mathcal{F}, \mu), p, c, \mathcal{G})$ where $\mathcal{G}$ is a $\sigma$-subfield of $\mathcal{F}$ and $p$ and $c$ are such that there exists $(\alpha, \beta) \in K(f)$ for which $p(\omega, x)=$ $\alpha(\omega) f(x)$ and $c(\omega, x)=\beta(\omega) x$ for all $(\omega, x) \in \Omega \times \mathbb{R}_{+}$. Each industry $I \in \mathcal{I}(f)$ is determined by a pair $(\alpha, \beta) \in K(f)$ and a $\sigma$-subfield $\mathcal{G}$ of $\mathcal{F}$, and can be described as $I=$ $((\Omega, \mathcal{F}, \mu), \alpha f, \beta, \mathcal{G})$. For every $(\alpha, \beta) \in K(f)$ we denote by $\left(q_{(\alpha, \beta)}, q_{(\alpha, \beta)}\right)$ the unique equilibrium of the Cournot game with symmetric information associated with the industry $I=((\Omega, \mathcal{F}, \mu), \alpha f, \beta, \mathcal{F})$, and by $\pi_{(\alpha, \beta)}=\alpha q_{(\alpha, \beta)} f\left(2 q_{(\alpha, \beta)}\right)-\beta q_{(\alpha, \beta)}$ the equilibrium profit. Also we define the function $U: K(f) \rightarrow \mathbb{R}$ by $U(\alpha, \beta)=E\left(\pi_{(\alpha, \beta)}\right)$. Clearly 
$U(\alpha, \beta)$ is well defined on $K(f)$. Define the set $M(f)=\left\{(x, y) \in \mathbb{R}_{++}^{2} \mid f(0) x>y\right\}$, a convex subset of $\mathbb{R}_{+}^{2}$, and let the function $V: M(f) \rightarrow \mathbb{R}$ be given by

$$
V(x, y)=U\left(x 1_{\Omega}, y 1_{\Omega}\right) .
$$

Note that $V$ is convex on $M(f)$ if and only if $U$ is convex on $K(f)$.

Remark 3.2. Let $(\alpha, \beta) \in K(f)$. For every $\sigma$-subfield $\mathcal{G}$ of $\mathcal{F}$ we have $(E(\alpha \mid \mathcal{G})$, $E(\beta \mid \mathcal{G})) \in K(f)$ and

$$
U(E(\alpha \mid \mathcal{G}), E(\beta \mid \mathcal{G}))=E\left(\pi_{\mathcal{G}}\right),
$$

where $\pi_{\mathcal{G}}$ is the firms' profit at the unique Bayesian equilibrium of the Cournot game with symmetric information associated with the industry $((\Omega, \mathcal{F}, \mu), E(\alpha \mid \mathcal{G}) f, E(\beta \mid \mathcal{G}), \mathcal{F})$.

Proof. Simply note that $(\alpha, \beta) \in K(f)$ implies $f(0) E(\alpha \mid \mathcal{G})(\omega)>E(\beta \mid \mathcal{G})(\omega)$ for all $\omega \in \Omega$. Therefore $(E(\alpha \mid \mathcal{G}), E(\beta \mid \mathcal{G})) \in K(f)$, and $U(E(\alpha \mid \mathcal{G}), E(\beta \mid \mathcal{G}))=E\left(\pi_{\mathcal{G}}\right)$.

Proposition 3.3 below is an analog of a well-known result in Blackwell's model. However, since the binary relation $\succeq$ defined in (3.2) contains Blackwell's ordering, the conclusion of Proposition 3.3 is stronger than that obtained in Blackwell's framework.

Proposition 3.3. Let $f: \mathbb{R}_{+} \rightarrow \mathbb{R}_{+}$be a function satisfying conditions (3.1.1)-(3.1.3) of Theorem 3.1. If the function $V$ defined in (3.5) is convex (concave) on $M(f)$, then the value of public information is positive (negative) in every symmetric duopolistic industry $I \in I(f)$.

Proof. Let $f: \mathbb{R}_{+} \rightarrow \mathbb{R}_{+}$be a function satisfying conditions (3.1.1)-(3.1.3) of Theorem 3.1, and assume that $V$ is convex on $M(f)$. (If $V$ is concave the proof is analogous.) Let $I \in \mathcal{I}(f)$. Thus $I=((\Omega, \mathcal{F}, \mu), \alpha f, \beta, \mathcal{G})$ for some $(\alpha, \beta) \in K(f)$ and some $\sigma$-subfield $\mathcal{G}$ of $\mathcal{F}$. Let $\mathcal{H}$ be a $\sigma$-subfield of $\mathcal{F}$ such that $\mathcal{H} \succeq \mathcal{G}$. By Remark 3.2, in order to prove that

$$
E\left(\pi_{\mathcal{H}}\right) \geqslant E\left(\pi_{\mathcal{G}}\right)
$$

we must show that

$$
U(E(\alpha \mid \mathcal{H}), E(\beta \mid \mathcal{H})) \geqslant U(E(\alpha \mid \mathcal{G}), E(\beta \mid \mathcal{G})) .
$$

By Theorem 34.4 in Billingsley (1995) we have

$$
E(\alpha \mid \mathcal{G})=E(E(\alpha \mid \mathcal{G} \vee \mathcal{H}) \mid \mathcal{G})
$$

and

$$
E(\beta \mid \mathcal{G})=E(E(\beta \mid \mathcal{G} \vee \mathcal{H}) \mid \mathcal{G})
$$

It is also easy to see that

$$
U\left(E\left(\alpha \mid \mathcal{F}^{\prime}\right), E\left(\beta \mid \mathcal{F}^{\prime}\right)\right)=E V\left(E\left(\alpha \mid \mathcal{F}^{\prime}\right), E\left(\beta \mid \mathcal{F}^{\prime}\right)\right),
$$

for every $\sigma$-subfield $\mathcal{F}^{\prime}$ of $\mathcal{F}$. Hence (3.6)-(3.8) imply 


$$
\begin{aligned}
U(E(\alpha \mid \mathcal{G}), E(\beta \mid \mathcal{G})) & =E V(E(\alpha \mid \mathcal{G}), E(\beta \mid \mathcal{G})) \\
& =E V(E(E(\alpha \mid \mathcal{G} \vee \mathcal{H}) \mid \mathcal{G}), E(E(\beta \mid \mathcal{G} \vee \mathcal{H}) \mid \mathcal{G}))
\end{aligned}
$$

And since $V$ is convex on $M(f)$, Jensen's Inequality implies

$$
\begin{aligned}
& E V(E(E(\alpha \mid \mathcal{G} \vee \mathcal{H}) \mid \mathcal{G}), E(E(\beta \mid \mathcal{G} \vee \mathcal{H}) \mid \mathcal{G})) \\
& \quad \leqslant E V(E(\alpha \mid \mathcal{G} \vee \mathcal{H}), E(\beta \mid \mathcal{G} \vee \mathcal{H})) .
\end{aligned}
$$

Since $\mathcal{H} \succeq \mathcal{G}$, then $E(\alpha \mid \mathcal{G} \vee \mathcal{H})=E(\alpha \mid \mathcal{H})$ and $E(\beta \mid \mathcal{G} \vee \mathcal{H})=E(\beta \mid \mathcal{H})$. Therefore (3.9) and (3.10) imply

$$
U(E(\alpha \mid \mathcal{G}), E(\beta \mid \mathcal{G})) \leqslant E V(E(\alpha \mid \mathcal{H}), E(\beta \mid \mathcal{H}))=U(E(\alpha \mid \mathcal{H}), E(\beta \mid \mathcal{H})) .
$$

In order to show the usefulness of Proposition 3.3 to determine the value of information in a symmetric duopolistic industry we present several applications.

Let $f: \mathbb{R}_{+} \rightarrow \mathbb{R}_{+}$be a function satisfying conditions (3.1.1)-(3.1.3) of Theorem 3.1. Denote by $\mathcal{I}_{0}(f)$ the class of industries of the form $I=\left((\Omega, \mathcal{F}, \mu), \alpha_{0} f, \beta, \mathcal{G}\right) \in \mathcal{I}(f)$, where $\alpha_{0}$ is a positive constant number. Thus, in every industry in the class $\mathcal{I}_{0}(f)$ the demand is known with certainty and only the cost is uncertain. Also denote by $\mathcal{I}_{1}(f)$ the class of industries of the form $I=\left((\Omega, \mathcal{F}, \mu), \alpha f, \beta_{1}, \mathcal{G}\right) \in \mathcal{I}(f)$, where $\beta_{1}$ is a positive constant number. In every industry in the class $\mathcal{I}_{1}(f)$ the cost is known with certainty and only the demand is uncertain. Let the function $V_{0}:(0, f(0)) \rightarrow \mathbb{R}$ be given for $y \in(0, f(0))$ by $V_{0}(y)=V(1, y)$. Also let the function $V_{1}:(1 / f(0), \infty) \rightarrow \mathbb{R}$ be given for $x \in(1 / f(0), \infty)$ by $V_{1}(x)=V(x, 1)$.

Proposition 3.4 provides a criterion for determining whether the value of public information is positive or negative for industries in the classes $\mathcal{I}_{0}(f)$ and $\mathcal{I}_{1}(f)$, respectively. The proof of Proposition 3.4 is analogous to that of Proposition 3.3 and is omitted.

Proposition 3.4. Let $f: \mathbb{R}_{+} \rightarrow \mathbb{R}_{+}$be a function satisfying conditions (3.1.1)-(3.1.3) of Theorem 3.1, and let $\mathcal{G}$ be a $\sigma$-subfield of $\mathcal{F}$.

(3.4.1) If $V_{0}$ is convex (concave) on $(0, f(0))$, then the value of public information is positive (negative) in every symmetric duopolistic industry $I \in \mathcal{I}_{0}(f)$.

(3.4.2) If $V_{1}$ is convex (concave) on $(1 / f(0), \infty)$, then the value of public information is positive (negative) in every symmetric duopolistic industry $I \in \mathcal{I}_{1}(f)$.

Our next proposition establishes that we can determine whether the function $V$ is convex (concave), by checking whether either $V_{0}$ or $V_{1}$ is convex (concave).

Proposition 3.5. Let $f: \mathbb{R}_{+} \rightarrow \mathbb{R}_{+}$be a function satisfying conditions (3.1.1)-(3.1.3) of Theorem 3.1, and assume that $f$ is twice continuously differentiable on $[0, \bar{x})$. The following conditions are equivalent: 
(3.5.1) $V$ is convex (concave) on $M(f)$;

(3.5.2) $V_{0}$ is convex (concave) on $(0, f(0))$;

(3.5.3) $V_{1}$ is convex (concave) on $(1 / f(0), \infty)$.

Proof. Proposition 3.5 follows from Lemma 3.6 below and the fact that $V$ is homogeneous of degree one.

Lemma 3.6. Let $f: \mathbb{R}_{+} \rightarrow \mathbb{R}_{+}$be a function satisfying conditions (3.1.1)-(3.1.3) of Theorem 3.1, and assume that $f$ is twice continuously differentiable on $[0, \bar{x})$. Then $\operatorname{sign}\left(V_{x x}\right)=\operatorname{sign}\left(V_{y y}\right)$. Moreover, if $V_{x x}(x, y) \geqslant 0\left(V_{x x}(x, y) \leqslant 0\right)$ for all $(x, y) \in M(f)$, then $V$ is convex (concave) on $M(f)$.

Proof. We show that for all $(x, y) \in M(f)$ the function $V: M(f) \rightarrow \mathbb{R}$ defined by (3.5) satisfies $V_{x x}(x, y) \geqslant 0$ if and only if $V_{y y}(x, y) \geqslant 0$. Let $(x, y) \in M(f)$ and let $\left(q_{(x, y)}, q_{(x, y)}\right)$ be the unique equilibrium of the industry $I=\left((\Omega, \mathcal{F}, \mu), x f, y 1_{\Omega}, \mathcal{F}\right)$, and write $Q(x, y)=2 q_{(x, y)}$. Then, for all $i \in\{1,2\}$ and $q_{i} \in S_{i}$ we have

$$
q_{(x, y)}(x f(Q(x, y))-y) \geqslant q_{i}\left(x f\left(q_{i}+q_{(x, y)}\right)-y\right) .
$$

Therefore, uniqueness of equilibrium implies

$$
Q(x, y)=Q\left(1, \frac{y}{x}\right)
$$

Also the first-order conditions for profits maximization imply

$$
\frac{1}{2} x Q(x, y) f^{\prime}(Q(x, y))+x f(Q(x, y))=y .
$$

Hence

$$
\begin{aligned}
V(x, y) & =-\frac{1}{4} x(Q(x, y))^{2} f^{\prime}(Q(x, y))=x\left(-\frac{1}{4}\left(Q\left(1, \frac{y}{x}\right)\right)^{2} f^{\prime}\left(Q\left(1, \frac{y}{x}\right)\right)\right) \\
& =x V\left(1, \frac{y}{x}\right) .
\end{aligned}
$$

Thus, for $\lambda>0$ we have

$$
V(\lambda x, \lambda y)=\lambda x V\left(1, \frac{\lambda y}{\lambda x}\right)=\lambda V(x, y)
$$

i.e., $V$ is homogeneous of degree one. By Euler's Theorem

$$
V(x, y)=x V_{x}(x, y)+y V_{y}(x, y)
$$

and therefore

$$
x V_{x x}(x, y)+y V_{y x}(x, y)=0 \quad \text { and } \quad y V_{y y}(x, y)+x V_{x y}(x, y)=0 .
$$

Thus $x^{2} V_{x x}(x, y)=y^{2} V_{y y}(x, y)$, and therefore $\operatorname{sign}\left(V_{x x}(x, y)\right)=\operatorname{sign}\left(V_{y y}(x, y)\right)$, and $V_{x x}(x, y) V_{y y}(x, y)-V_{x y}(x, y) V_{y x}(x, y)=0$. Since the eigenvalues of the Hessian matrix of $V$ at $(x, y)$ are 0 and $V_{x x}(x, y)+V_{y y}(x, y)$, then $V_{x x}(x, y) \geqslant 0\left(V_{x x}(x, y) \leqslant 0\right)$ for all $(x, y) \in M(f)$ implies that $V$ is convex (concave) on $M(f)$. 
The following remark is a direct implication of Proposition 3.5.

Remark 3.7. If the value of public information in every industry $I \in \mathcal{I}_{0}(f)$ (or $I \in \mathcal{I}_{1}(f)$ ) is positive (negative), then the value of public information of every industry $I \in \mathcal{I}(f)$ is positive (negative).

Theorem 3.8 establishes useful conditions under which the value of information is positive in an industry. These conditions are essentially the same that Novshek (1985) imposes to guarantee existence of a Cournot equilibrium. (Condition (3.8.1) is the main condition of Novshek's Theorem 3.)

Theorem 3.8. Let $f: \mathbb{R}_{+} \rightarrow \mathbb{R}_{+}$be a function satisfying conditions (3.1.1)-(3.1.3) of Theorem 3.1. If $f$ is thrice differentiable on $[0, \bar{x})$ and for all $x \in[0, \bar{x})$ satisfies

(3.8.1) $x f^{\prime \prime}(x)+f^{\prime}(x) \leqslant 0$ and

(3.8.2) $f^{\prime \prime \prime}(x) \leqslant 0$,

then the value of public information in any industry $I \in \mathcal{I}(f)$ is positive.

Proof. Let $f$ be a function satisfying the assumptions of Theorem 3.8 and let $I \in \mathcal{I}(f)$. By Proposition 3.5 we may assume, without loss of generality, that $I \in \mathcal{I}_{0}(f)$. Thus the industry $I$ is described by a collection $I=\left((\Omega, \mathcal{F}, \mu), f, y 1_{\Omega}, \mathcal{G}\right)$, for some $0<y<f(0)$. We show that $V_{0}^{\prime \prime}(y)=V_{y y}(1, y) \geqslant 0$ for $0<y<f(0)$, and therefore that $V_{0}$ is convex on $(0, f(0))$.

Let $0<y<f(0)$, and let $\left(q_{y}, q_{y}\right)$ be the unique Bayesian equilibrium of the Cournot game associated with $I$. Write $Q(y)=2 q_{y}$. We have

$$
V(1, y)=\frac{Q(y)}{2}(f(Q(y))-y)
$$

First-order conditions for profit maximization imply

$$
\frac{Q(y)}{2} f^{\prime}(Q(y))+f(Q(y))=y .
$$

Therefore

$$
V(1, y)=\frac{-Q^{2}(y)}{4} f^{\prime}(Q(y)) .
$$

By (3.8.1) $Q(y)$ is the unique solution to the equation

$$
\frac{z}{2} f^{\prime}(z)+f(z)=y \text {. }
$$

By the Implicit Function Theorem, $Q$ is differentiable on $(0, f(0))$. Thus, differentiating (3.11) we get

$$
Q^{\prime}(y)=\frac{2}{Q(y) f^{\prime \prime}(Q(y))+3 f^{\prime}(Q(y))} .
$$


Direct calculation yields

$$
V_{0}^{\prime \prime}(y)=\frac{-f^{\prime}(Q(y))\left(Q^{2}(y) f^{\prime \prime \prime}(Q(y))+6 Q(y) f^{\prime \prime}(Q(y))+6 f^{\prime}(Q(y))\right)}{2\left(Q(y) f^{\prime \prime}(Q(y))+3 f^{\prime}(Q(y))\right)^{3}} .
$$

Since $f^{\prime}<0$ on $[0, \bar{x}),(3.8 .1)$ and (3.8.2) imply $V_{0}^{\prime \prime}(y) \geqslant 0$. Therefore $V_{0}$ is convex on $(0, f(0))$, and by Propositions 3.4 and 3.5 the value of public information in every industry $I \in \mathcal{I}(f)$ is positive.

The following result is a direct corollary of Theorem 3.8.

Corollary 3.9. Let $f: \mathbb{R}_{+} \rightarrow \mathbb{R}_{+}$be a function satisfying conditions (3.1.1)-(3.1.3) of Theorem 3.1. If $f$ is concave, thrice differentiable on $[0, \bar{x})$ and satisfies $f^{\prime \prime \prime}(x) \leqslant 0$ for $0 \leqslant x<\bar{x}$, then the value of public information in any industry $I \in \mathcal{I}(f)$ is positive.

We now apply our results to some examples.

Example 3.10. Let $f$ be given for $x \in \mathbb{R}_{+}$by

$$
f(x)=\max \left\{a_{0}-\sum_{i=1}^{n} a_{i} x^{\lambda_{i}}, 0\right\}
$$

where $a_{0}>0, a_{i} \geqslant 0$, and $\lambda_{i} \geqslant 1$ for $i \in\{1, \ldots, n\}$. The function $f$ satisfies conditions (3.1.1)-(3.1.3) of Theorem 3.1. Let $\mathcal{G}$ be a $\sigma$-subfield of $\mathcal{F}$, and for $0<y<a_{0}=f(0)$ let $(q(y), q(y))$ be the unique Bayesian equilibrium of the Cournot game associated with the industry $I=\left((\Omega, \mathcal{F}, \mu), f, y 1_{\Omega}, \mathcal{G}\right)$. Write $Q(y)=2 q(y)$. Now, we have

$$
x^{2} f^{\prime \prime \prime}(x)+6 x f^{\prime \prime}(x)+6 f^{\prime}(x)=\sum_{i=1}^{n}\left(\lambda_{i}^{2}+3 \lambda_{i}+2\right) a_{i} x^{\lambda_{i}-1}<0
$$

and

$$
x f^{\prime \prime}(x)+3 f^{\prime}(x)=-\sum_{i=1}^{n} \lambda_{i}\left(\lambda_{i}+4\right) a_{i} x^{\lambda_{i}-1}<0 .
$$

Since $f^{\prime}(x)<0$ for all $x>0,(3.12)$ yields $V_{0}^{\prime \prime}(y)>0$ for all $0<y<a_{0}=f(0)$. Thus, $V_{0}$ is convex on $\left(0, a_{0}\right)$, and therefore by Propositions 3.4 and 3.5 the value of information in any industry $I \in \mathcal{I}(f)$ is positive.

Example 3.11. Let $f$ be given for $x \in \mathbb{R}_{+}$by

$$
f(x)= \begin{cases}(x-a)^{2} & \text { if } 0 \leqslant x \leqslant a, \\ 0 & \text { otherwise }\end{cases}
$$

where $a>0$. The function $f$ satisfies conditions (3.1.1)-(3.1.3) of Theorem 3.1. Let $\mathcal{G}$ be a $\sigma$-subfield of $\mathcal{F}$, and for $0<y<f(0)=a^{2}$ let $(q(y), q(y))$ be the unique Bayesian equilibrium of the Cournot game associated with the industry $I=\left((\Omega, \mathcal{F}, \mu), f, y 1_{\Omega}, \mathcal{G}\right)$. 
Write $Q(y)=2 q(y)$. It is easy to check that the first-order conditions for profit maximization yield $0 \leqslant Q(y) \leqslant a / 2$ for all $0<y<a^{2}$. Now, for all $0<y<a^{2}$ we have

$$
Q(y) f^{\prime \prime}(Q(y))+f^{\prime}(Q(y))=4 Q(y)-2 a \leqslant 0 \quad \text { and } \quad 0=f^{\prime \prime \prime}(Q(y)) .
$$

Therefore by (3.12) we have $V_{0}^{\prime \prime}(y)>0$ for all $0<y<f(0)$. Thus, $V_{0}$ is convex on $(0, f(0))$, and therefore by Propositions 3.4 and 3.5 the value of public information in any industry $I \in \mathcal{I}(f)$ is positive.

Example 3.12. Let $f$ be given for $x \in \mathbb{R}_{+}$by

$$
f(x)= \begin{cases}(1-x)^{3} & \text { if } 0 \leqslant x \leqslant 1, \\ 0 & \text { otherwise. }\end{cases}
$$

The function $f$ satisfies conditions (3.1.1)-(3.1.3) of Theorem 3.1 on [0,1). Let $\mathcal{G}$ be a $\sigma$-subfield of $\mathcal{F}$, and for $0<y<1=f(0)$ let $(q(y), q(y))$ be the unique Bayesian equilibrium of the Cournot game associated with the industry $I=\left((\Omega, \mathcal{F}, \mu), f, y 1_{\Omega}, \mathcal{G}\right)$. Write $Q(y)=2 q(y)$. It is easy to check that the first-order conditions for profit maximization imply $0 \leqslant Q(y) \leqslant 2 / 5$ for all $0<y<1$. Direct computation yields

$$
Q(y) f^{\prime \prime}(Q(y))+3 f^{\prime}(Q(y))=(1-Q(y))(15 Q(y)-9)<0
$$

and

$$
Q^{2}(y) f^{\prime \prime \prime}(Q(y))+6 Q(y) f^{\prime \prime}(Q(y))+6 f^{\prime}(Q(y))=-60 Q^{2}(y)+72 Q(y)-18,
$$

for all $0<y<1$. Now, when $y$ approaches $0, Q(y)$ approaches $2 / 5$, and thus the above expression is negative. Therefore by (3.12) we have $V_{0}^{\prime \prime}(y)<0$ for all $0<y<\bar{y}$, where $\bar{y}>0$ is sufficiently small that the above expression is negative. Thus, $V_{0}$ is concave on $(0, \bar{y})$, and therefore there exists a subclass $\mathcal{J}(f)$ of $\mathcal{I}(f)$ such that for any industry $I \in \mathcal{J}(f)$ the value of public information is negative.

\section{Extensions: asymmetric information}

We conclude the paper with an example that illustrates the difficulties that emerge when firms are asymmetrically informed. In this example the Cournot game associated with the given industry has a unique interior Bayesian equilibrium, but the game obtained by adding some public information has a unique corner equilibrium.

Example 4.1. Let $(\Omega, \mathcal{F}, \mu)$ be a probability space, where $\Omega=\left\{\omega_{1}, \omega_{2}, \omega_{3}\right\}, \mathcal{F}=2^{\Omega}$, and $\mu\left(\omega_{1}\right)=1 / 2, \mu\left(\omega_{2}\right)=3 / 8, \mu\left(\omega_{3}\right)=1 / 8$. Define $p: \Omega \times \mathbb{R}_{+} \rightarrow \mathbb{R}$ for $(\omega, Q) \in \Omega \times \mathbb{R}_{+}$ by

$$
p(\omega, Q)= \begin{cases}80-Q & \text { if } Q \leqslant 80 \\ 0 & \text { otherwise. }\end{cases}
$$

Also let $c: \Omega \times \mathbb{R}_{+} \rightarrow \mathbb{R}$ be given for $(\omega, Q) \in \Omega \times \mathbb{R}_{+}$by $c(\omega, Q)=\beta(\omega) Q$, where

$$
\beta(\omega)= \begin{cases}20 & \text { if } \omega=\omega_{1}, \\ 1 & \text { if } \omega=\omega_{2}, \\ 77 & \text { if } \omega=\omega_{3} .\end{cases}
$$


Let $\mathcal{F}_{1}=\left\{\emptyset, \Omega,\left\{\omega_{1}\right\},\left\{\omega_{2}, \omega_{3}\right\}\right\}, \mathcal{F}_{2}=\{\emptyset, \Omega\}$, and let $\mathcal{G}=\left\{\emptyset, \Omega,\left\{\omega_{1}, \omega_{3}\right\},\left\{\omega_{2}\right\}\right\}$. Then $\mathcal{F}_{1} \vee \mathcal{G}=2^{\Omega}$, and $\mathcal{F}_{2} \vee \mathcal{G}=\mathcal{G}$. The unique Bayesian equilibrium of the Cournot game associated with the industry $\left((\Omega, \mathcal{F}, \mu), p, c, \mathcal{F}_{1}, \mathcal{F}_{2}\right)$ is $\left(q_{1}, q_{2}\right)$ where $q_{1}(\omega)=$ $q_{2}(\omega)=20$ for all $\omega \in \Omega$. Also the Cournot game associated with the industry $\left((\Omega, \mathcal{F}, \mu), p, c, \mathcal{F}_{1} \vee \mathcal{G}, \mathcal{F}_{2} \vee \mathcal{G}\right)$, has a unique a Bayesian equilibrium, $\left(\hat{q}_{1}, \hat{q}_{2}\right)$. In this equilibrium we have $\hat{q}_{1}\left(\omega_{3}\right)=0$, and therefore it is a "corner" equilibrium.

\section{Acknowledgments}

We thank Ori Haimanko for helpful discussions. The research of Einy and Shitovitz was supported by The Israel Science Foundation, grant 871/01-31. Moreno acknowledges the support of the Ministerio de Ciencia y Tecnología, grant BEC2000-0173.

\section{References}

Amir, R., 1996. Cournot oligopoly and the theory of supermodular games. Games Econ. Behav. 15, 132-148.

Aumann, R.J., 1969. Measurable utility and the measurable choice theorem. In: Colloq. Int. CNRS, Vol. 171, pp. 15-26.

Blackwell, D., 1951. Comparison of experiments. In: Neymann, J. (Ed.), Proceeding of the Second Berkeley Symposium on Mathematical Statistics and Probability. Univ. of California Press.

Billingsley, P., 1995. Probability and Measure. Wiley, New York.

Einy, E., Haimanko, O., Orzach, A., Sela, A., 2002. Dominant strategies, superior information, and winner's curse in second price auctions. Int. J. Game Theory 30, 405-419.

Einy, E., Moreno, D., Shitovitz, B., 2002. Information advantage in Cournot oligopoly. J. Econ. Theory 106, $151-160$.

Hildenbrand, W., 1974. Core and Equilibria in Large Economies. Princeton Univ. Press, Princeton.

Hirshleifer, J., 1971. The private and social value of information and reward to incentive activity. Amer. Econ. Rev. 61, 561-574.

Gal-Or, E., 1985. Information sharing in oligopoly. Econometrica 53, 329-343.

Gal-Or, E., 1986. Information transmission-Cournot and Bertrand equilibria. Rev. Econ. Stud. 53, 85-92.

Green, J., 1981. The value of information with sequential future markets. Econometrica 49, 335-358.

Laffont, J.-J., 1989. The Economics of Uncertainty and Information. MIT Press, Cambridge, MA.

Marshall, J., 1979. Private incentives and public information. Amer. Econ. Rev. 64, 373-390.

Novshek, W., 1985. On the existence of Cournot equilibrium. Rev. Econ. Stud. 52, 85-98.

Ottaviani, M., Pratt, A., 2001. The value of public information in monopoly. Econometrica 69, 1673-1683.

Ponssard, J., 1979. The strategic role of information on demand function in an oligopolistic market. Manage. Sci. 25, 243-250.

Schlee, E., 2001. The value of information in efficient risk-sharing arrangements. Amer. Econ. Rev. 91, 509-524. Sakai, Y., 1985. The value of information in a simple duopoly model. J. Econ. Theory 36, 36-54.

Sulganik, E., Zilcha, I., 1996. The value of information in the presence of future markets. J. Future Markets 16, $227-240$.

Vives, X., 1984. Duopoly information equilibrium: Cournot and Bertrand. J. Econ. Theory 34, 71-94.

Wilson, R., 1975. Informational economies of scale. Bell J. Econ. 6, 184-195. 